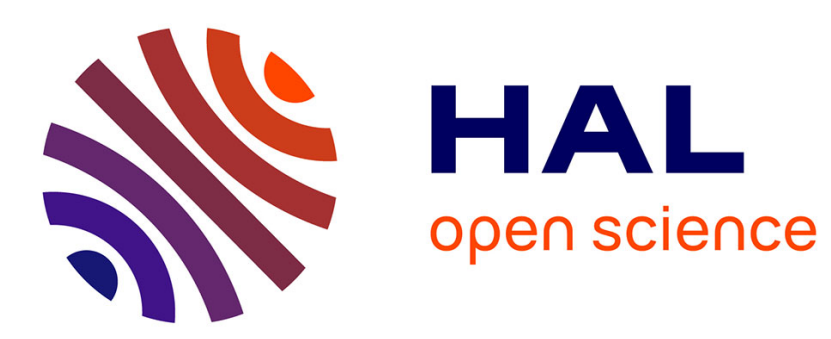

\title{
Lipschitz-Killing curvatures of the Excursion Sets of Skew Student's t Random fields
}

Ola Suleiman Ahmad, Jean-Charles Pinoli

\section{To cite this version:}

Ola Suleiman Ahmad, Jean-Charles Pinoli. Lipschitz-Killing curvatures of the Excursion Sets of Skew Student's t Random fields. Stochastic Model, 2013, 29 (2), pp.273-289. 10.1080/15326349.2013.783290 . hal-00834849

\section{HAL Id: hal-00834849 https://hal.science/hal-00834849}

Submitted on 3 Jul 2013

HAL is a multi-disciplinary open access archive for the deposit and dissemination of scientific research documents, whether they are published or not. The documents may come from teaching and research institutions in France or abroad, or from public or private research centers.
L'archive ouverte pluridisciplinaire HAL, est destinée au dépôt et à la diffusion de documents scientifiques de niveau recherche, publiés ou non, émanant des établissements d'enseignement et de recherche français ou étrangers, des laboratoires publics ou privés. 


\title{
LIPSCHITZ-KILLING CURVATURES OF THE EXCURSION SETS OF SKEW STUDENT'S $\boldsymbol{t}$ RANDOM FIELDS
}

\section{Ola Suleiman Ahmad and Jean-Charles Pinoli}

\author{
École Nationale Supérieure des Mines de Sainl-Étienne, Sainl-Élienne, France
}

\begin{abstract}
$\square \quad$ In many applications related with geoslatistics, biological and medical imaging, material science, and engineering surfaces, the real observations have asymmetric and heavy-lailed multivariate distributions. These observations are spatially correlated and they could be modeled by the skew random fields. However, several statistical analysis problems require studying the integral geometry of these random fields in order to detect the local changes between the different samples, via the expected Euler-Poincaré characteristic and the intrinsic volumes (LipschitzKilling curvatures) of the excursion set obtained at a given threshold. This article is interested in a class of skew random fields, namely, skew Students $t$ random field. The goal is to derive an explicit formula of Lipschitz-Killing curvatures and the expected Euler-Poincare characteristic of the skew Student s $t$ excursion sets on a compact subset $S$ of $\mathbb{R}^{2}$ by extending previous resulls reported in the literature. The motivation comes from the need to model the roughness of some engineering surfaces in order to delect the local changes of the surface peaks/valleys during a specific physical phenomenon. The analytical and empirical Euler-Poincaré characteristics are compared in order to test the skew Students $t$ random field on the real surface. Simulation results are also presented in the article for illustration and validation.
\end{abstract}

Keywords Excursion sets; Expected Euler-Poincaré characteristic; Lipschitz-Killing curvatures; Skewness; Skew Student's $t$ random field; Surface roughness.

Mathematics Subject Classification Primary 60D05; Secondary 60G60, 52A22.

\section{INTRODUCTION}

In a wide range of real applications, there is a great need to model observations that have asymmetric behavior and heavy-tailed univariate or multivariate distributions. Among the most used models that are developed for this aim, are the skew Gaussian and skew Student's $t$

Received August 2012; Accepted November 2012

Address correspondence to Ola Suleiman Ahmad, École Nationale Supérieure des Mines de SaintÉtienne, LGF, UMR CNRS 5307, 158 cours Fauriel, Saint-Étienne 42023, France; E-mail: olasahmad@ gmail.com 
families. ${ }^{\mid 7-11,15,16]}$ In many statistical problems, the random observations are spatially correlated, and the best solution is to represent using the theory of random fields. ${ }^{[1,2,23]}$ The shape of the sample's distribution, skewness and kurtosis, is of special interest since its changes could interpret certain physical and mechanical phenomenon, as well as capture certain anomalies or activations inside the region of interest that represents the random data. Such kinds of samples cannot be modeled by either Gaussian random fields or skew Gaussian random fields. ${ }^{[6,10,11]}$ Skew Student's $t$ random fields arise among the flexible class of stochastic models that could be used for such real problems. They are characterized by the skewness, which describes the asymmetry behavior of the sample's distribution, and the degree of freedom, which controls the heavy-tailed shape of the probability density function.

The motivation to study this class of skew random fields is due to the need to model the roughness topography of certain engineering surfaces during the wear process; e.g., surfaces involved into hip joint replacement. The surface topography is represented on a subset $S \subset \mathbb{R}^{2}$ as a $3 \mathrm{D}$ map of correlated heights distributed with respect to a skew Student's $t$ random field. One of the interesting issues to describe the roughness of the surface is the evaluation of the number of peaks and valleys, which is related to the local maxima and minima of the skew Student's $t$ random field.

The local maxima or minima indicate the local changes inferred by the probability that a random field, $Y$, exceeds a high threshold $h$ in $S$. The regions of the local changes define the excursion set of the random field $Y$ above $h$. Furthermore, the geometric properties of the excursion sets are of great interest in a wide range of applications, since they have a physical interpretation of many phenomenons.

The integral geometry ${ }^{[13,18]}$, provides interesting characteristic functions that could be used to measure the $N$-dimensional volumes of the excursion sets of a large class of random fields (e.g., area, boundary length, and Euler-Poincaré characteristic for $N=2$ ), namely, Minkowski functionals or Lipschitz-Killing curvatures. ${ }^{15,19]}$ Euler-Poincaré characteristic counts, in two dimensions, the number of connected components, minus the number of holes of the excursion set, when it does not touch the boundaries of the subset $S{ }^{[22]}$ At high thresholds, the Euler-Poincaré characteristic counts only the number of connected components (i.e., isolated peaks) inside the excursion set. Hence, it is considered as an accurate approximation of the probability that the maxima of the random field will exceed those thresholds. ${ }^{[2,5]}$

This article considers a class of skew random fields, called skew Student's $t$ random fields with $v$ degrees of freedom (Section 4). This 
work focuses on introducing this random field on a subset $S \subset \mathbb{R}^{2}$ and on deriving the analytical formulae of the Lipschitz-Killing curvatures of its excursion sets and the expected Euler-Poincaré characteristic (Section 5). Simulation results are reported (Section 6) for several realizations of a stationary skew Student's $t$ random field for validation. The skew Student's $l$ random field has been tested on the roughness topography of a real engineering surface using the expected Euler-Poincaré characteristic (Section 7).

\section{PRELIMINARIES}

A real-valued random field, denoted by $\{Y=Y(x): x \in S\}$, is defined on a nonempty compact subset $S \subset \mathbb{R}^{2}$, with mean $\mu_{Y}$ and variance $\sigma_{Y}^{2} . Y$ will be supposed stationary but not necessarily isotropic. The probability density function of $Y$ is denoted by $p_{Y}$ and its cumulative distribution function is denoted by $P_{Y}$.

\section{THE SKEW STUDENT'S $\boldsymbol{t}$ DISTRIBUTION}

This section briefly reviews the skew Student's $t$ distribution, ${ }^{[8,9,11,15]}$ before defining the skew Student's $\ell$ random field.

\subsection{Univariate Skew Student's $t$ Distribution}

Let us consider a skew Student's $t$ random variable $Y$ with $v$ degrees of freedom, skewness index $\alpha$, mean value $\mu_{Y}$, and variance $\sigma_{Y}^{2}$. Then, the probability density function of $Y$ is defined as:

$$
p_{Y}(h ; \alpha, v)=\frac{2}{\sigma_{y}} t_{1}(u ; v) T_{1}\left(\alpha u \sqrt{\frac{v+1}{v+u^{2}}} ; v+1\right),
$$

where $u=\left(y-\mu_{Y}\right) / \sigma_{Y}, t_{1}(. ; v)$ is the standard Student's $t$ distribution with $v$ degrees of freedom, and $T_{1}$ is the Student's $t$ cumulative distribution function with $v+1$ degrees of freedom. The real parameter $\alpha$ controls the skewness. For $\alpha>0$ (resp. $\alpha<0$ ) the distribution has a positive (resp. negative) skewness. When $\alpha=0$ the distribution is symmetric and it turns back to the known Student's $t$ distribution.

The stochastic representation of the skew Student's $t$ random variable $Y$, which has the probability density function defined in (3.1), is given by:

$$
Y=\mu_{Y}+\sigma_{Y} V^{-1 / 2} Z
$$


where $V \sim \chi_{v}^{2} / v$ is the chi-squared random variable independent of $Z$, and $Z$ is the known skew Gaussian random variable ${ }^{\mid 71}$ defined as:

$$
Z=\delta|X|+\sqrt{1-\delta^{2}} G,
$$

where $X, G \sim \operatorname{Normal}_{1}(0,1)$ are independent normal random variables, and $\delta=\alpha / \sqrt{1+\alpha^{2}}$.

\subsection{Multivariate Skew Student's $t$ Distribution}

The $d$-variate skew Student's $t$ distribution with $v$ degrees of freedom for a random vector $\boldsymbol{Y}=\left(Y_{1}, \ldots, Y_{d}\right)^{t}$ has the following probability density function:

$$
\begin{aligned}
& p_{Y}\left(y ; \boldsymbol{\mu}_{\boldsymbol{Y}}, \boldsymbol{\alpha}, \boldsymbol{\Sigma}, v\right) \\
& \quad=2 l_{d}\left(y ; \boldsymbol{\mu}_{Y}, \boldsymbol{\Sigma}, v\right) \\
& \quad \times T_{1}\left(\boldsymbol{\alpha}^{t} \Sigma^{-1 / 2}\left(y-\mu_{Y}\right) \sqrt{\frac{v+d}{v+\left(y-\mu_{Y}\right)^{t} \Sigma^{-1}\left(y-\mu_{Y}\right)}} ; v+d\right),
\end{aligned}
$$

where $t_{p}\left(. ; \mu_{Y}, \Sigma, v\right)$ is the density of the $d$-variate Student's $t$ distribution with $v$ degrees of freedom, $(d \times d)$ covariance matrix $\Sigma$, and $d$-dimensions mean vector $\mu_{Y} . T_{1}(. ; v+d)$ is the scalar cumulative distribution of the standard Student's $t$ distribution with $v+d$ degrees of freedom, and $\alpha$ refers to the skewness vector.

\section{SKEW STUDENT'S $\boldsymbol{t}$ RANDOM FIELD}

The skew Student's $t$ random field introduced in this article is restricted to be defined on a nonempty compact subset $S \subset \mathbb{R}^{2}$.

In order to introduce the skew Student's $t$ random field, let us recall the definition of the Student's $t$ random field, ${ }^{[21]}$ :

Definition 4.1. Let $G_{1}(x), \ldots, G_{v+1}(x), x \in S$, be independent, identically distributed, homogeneous, real-valued Gaussian random fields with zero mean, unit variance, and $\Lambda=\operatorname{Var}\left(\partial G_{i} / \partial x\right), i=1, \ldots, v+1$. Then, the Student's $l$ random field with $v$ degrees of freedom, denoted $T^{v}(x)$, is defined as:

$$
T^{v}(x)=\frac{\sqrt{v} G_{1}(x)}{\sqrt{\sum_{k=2}^{v+1} G_{k}^{2}(x)}}
$$


The following skew Student's $t$ random field extends the definition of the Student's $l$ random field.

Definition 4.2. Let $U$ be a stationary Student's $t$ random field with $v$ degrees of freedom defined as above. Let $U_{0}(x)$ be defined such that:

$$
U_{0}(x)=\frac{\sqrt{v} z}{\sqrt{\sum_{k=2}^{v+1} G_{k}^{2}(x)}}
$$

where $z \sim \operatorname{Normal}(0,1)$ is a standard Gaussian random variable. A stationary random field, $Y$, with zero mean, unit variance $\left(\sigma_{y}^{2}=1\right)$, and $\Lambda=\operatorname{Var}\left(\partial G_{i} / \partial x\right), i=1, \ldots, v+1$ at any fixed point $x \in S$ given by:

$$
Y(x)=\delta U_{0}(x)+\sqrt{1-\delta^{2}} U(x)
$$

with a skewness index $\delta,(\delta<1)$, defines a stationary skew Student's $t$ random field with $v$ degrees of freedom.

Where the family of all finite-dimensional distributions of $Y$ are multivariate skew Student's $t$ distributions, and the marginal distribution of $Y$ at any fixed $x$ is a skew Student's $t$ distribution with $v$ degrees of freedom and skewness index $\delta, p_{Y}$.

In the next section, we will focus on the geometry of the excursion sets of the skew Student's $t$ random field defined in (4.3), precisely on the expectation of their Lipschitz-Killing curvatures.

\section{LIPSCHITZ-KILLING CURVATURES OF THE SKEW STUDENT'S $t$ EXCURSION SETS}

\subsection{Theory}

The direct way to study the geometry of the skew Student's $t$ excursion sets is by Minkowski functionals, or the so-called Lipschitz-Killing curvatures, for the non-isotropic random fields. ${ }^{\mid 19]}$ The excursion set of a random field $Y$, defined on a nonempty compact subset $S \subset \mathbb{R}^{d}, d \geq 1$, is the set of all points in $S$ where the random field exceeds a threshold $h$. They are expressed as ${ }^{[2,3]}$ :

$$
E_{h} \equiv E_{h}(Y, S)=\{x \in S, Y(x) \geq h\}
$$

The Student's $t$ random field $T^{v}(x)$ in (4.1) is defined in terms of stationary independent and identically distributed (i.i.d.) Gaussian random fields, $G_{i}(x),(i=1, \ldots, v+1)$, with zero mean, unit variance, and the 
$(d \times d)$ matrix, $\Lambda$, of their second spectral moments $\lambda_{k l},(l, k=1, \ldots, d)$ whose elements are

$$
\lambda_{k l}=\mathbb{E}\left[\frac{\partial G_{i}}{\partial x_{l}} \frac{\partial G_{i}}{\partial x_{k}}\right]
$$

for all $G_{i}(i=1, \ldots, v+1)$.

The mean $j$ th dimensional Lipschitz-Killing curvatures are defined for any random field, expressed in terms of i.i.d. nonisotropic Gaussian random fields, from the basic Gaussian kinematic formulae, ${ }^{[19,20]}$ such that:

$$
\mathbb{E}\left[\mathscr{L}_{j}\left(E_{h}(Y, S)\right)\right]=\sum_{k=0}^{d-j}\left[\begin{array}{c}
j+k \\
k
\end{array}\right] \mathscr{L}_{k}(S) \rho_{k}(h),
$$

where $\left[\begin{array}{c}j+k \\ k\end{array}\right]$ is a flag coefficient $\mathscr{L}_{k}(S),(k=1, \ldots, d)$, defines the $k$ th dimensional Lipschitz-Killing curvatures of $S$. The functions $\rho_{k}(h)$ are the $k$ th dimensional Euler characteristic (EC) densities for the random field $Y$, in our case the skew Student's $t$ random field, and they do not depend neither on the geometry of $S$ or on the matrix $\Lambda$.

The Lipschitz-Killing curvatures, $\mathscr{L}_{k}(S)$, of $S$ measure its intrinsic volumes in the Riemannian metric defined by the variogram of the first-order partial derivatives of the Gaussian components. This means replacing the local Euclidean distance between any two points of $S$ by $\operatorname{det}(\Lambda)^{1 / 2}$. In the case $k=d$, the $d$-dimensional Lipschitz-Killing curvatures $\mathscr{L}_{d}(S)$ of $S$ defines the $d$ th volume of $S$ such that:

$$
\mathscr{L}_{d}(S)=\int_{S} \operatorname{det}\left(\Lambda_{x}\right)^{1 / 2} d x
$$

and for $k=d-1$, the $(d-1)$-dimensional Lipschitz-Killing curvature of $S$ is

$$
\mathscr{L}_{d-1}(S)=\frac{1}{2} \int_{\partial S} \operatorname{det}\left(\Lambda_{x}\right)^{1 / 2} d x,
$$

where $\Lambda_{x}$ is the local version of $\Lambda,^{[5]}$ computed at each point $x \in S$.

When $k=0, \mathscr{L}_{0}(S)$ is the Euler-Poincaré characteristic of $S$.

The expected Euler-Poincaré characteristic of the excursion set $E_{h}(Y, S)$ is then defined when $j=0$ such that $\mathbb{E}\left[\chi\left(E_{h}(Y, S)\right)\right]=$ $\mathbb{E}\left[\mathscr{L}_{0}\left(E_{h}(Y, S)\right)\right]$. At high thresholds, it estimates the number of the maxima of $Y^{[2]}$ :

$$
\mathbb{P}\left[\sup _{x \in S} Y(x) \geq h\right] \cong \mathbb{E}\left[\chi\left(E_{h}(Y, S)\right)\right], \quad(h \rightarrow \infty)
$$

For $j>0, \mathscr{L}_{j}\left(E_{h}(Y, S)\right)$ measures the $j$ th size of the excursion set at the level $h$. In $\mathbb{R}^{2}, \mathscr{L}_{1}\left(E_{h}(Y, S)\right)$ is the half-boundary length, and $\mathscr{L}_{2}\left(E_{h}(Y, S)\right)$ 
is the area of $E_{h}$. In $\mathbb{R}^{3}, \mathscr{L}_{3}\left(E_{h}(Y, S)\right)$ is the volume, $\mathscr{L}_{2}\left(E_{h}(Y, S)\right)$ is half the surface area, and $\mathscr{L}_{1}\left(E_{h}(Y, S)\right)$ is the integral mean curvature of the excursion set $E_{l l}$.

\subsection{Notation}

In this article, the skew Student's $t$ random field is considered stationary over the two-dimensional subset, $S$, which is defined as a rectangle $\left[0, R_{1}\right] \times\left[0, R_{2}\right], R_{i} \in \mathbb{R}^{+}$, in $\mathbb{R}^{2}$. Then, the Lipschitz-Killing curvatures of $S, \mathscr{L}_{k}(S)$, for a stationary skew Student's $t$ random field with respect to the Riemannian metric in $\mathbb{R}^{2}$, are

$$
\begin{aligned}
& \chi(S)=\mathscr{L}_{0}(S)=1, \\
& C(S)=\mathscr{L}_{1}(S)=R_{1} \lambda_{11}^{1 / 2}+R_{2} \lambda_{22}^{1 / 2}, \\
& A(S)=\mathscr{L}_{2}(S)=R_{1} R_{2} \times \operatorname{det}(\Lambda)^{1 / 2},
\end{aligned}
$$

where $\mathscr{L}_{0}(S)$ is the Euler-Poincaré characteristic of $S$, denoted by $\chi(S)$; $\mathscr{L}_{1}(S)$ is half the boundary length of $S$, denoted by $C(S)$; and $\mathscr{L}_{2}(S)$ is the two-dimensional area of $S$, denoted by $A(S)$.

\subsection{Expectations}

The $k$ th dimensional EC density functions of the skew Student's $t$ random field, $Y(x)$, could be calculated using Morse theory. ${ }^{[1]}$ They are expressed for any twice-differentiable and isotropic random field ${ }^{[5,19,21]}$ as follows:

$$
\begin{aligned}
\rho_{k}(h) & =\mathbb{E}\left(1_{(Y \geq h)} \operatorname{det}\left(-\ddot{Y}_{\mid k}\right) \dot{Y}_{\mid k}=0\right) \mathbb{P}\left(\dot{Y}_{\mid k}=0\right) \\
& =\mathbb{E}\left(\dot{Y}_{k}^{+} \operatorname{det}\left(-\ddot{Y}_{k-1}\right) \dot{Y}_{k-1}=0, Y=h\right) p_{k-1}(0, h),
\end{aligned}
$$

where $\dot{Y}_{\mid k-1}$ and $\ddot{Y}_{k-1}$ are the first- and second-order partial derivatives of the first $(k-1)$ th elements of $Y(x)$ at any point $x \in S$ (i.e., the $(k-$ 1) $\times(k-1)$ submatrix of $\dot{Y}$ and $\ddot{Y}$, respectively). $p_{k-1}(0, h)$ is the joint probability density function of $\dot{Y}_{k-1}$ and $Y$, whereas $\dot{Y}_{j}^{+}=\max \left(0, \dot{Y}_{j}\right)$.

Proposition 5.3.1. The $k$-th dimensional EC densities, $\rho_{k}(),. j=0,1,2$, of a slalionary skew Sludenl $s$ l random field, $Y$, wilh $v$ degrees of freedom and skewness index $\delta(\delta<1)$ on $\mathbb{R}^{2}$ for a given threshold $h$ are expressed as:

(i) $\quad \rho_{0}(h)=2 \frac{\Gamma\left(\frac{v+1}{2}\right)}{\sqrt{v \pi} \Gamma\left(\frac{v}{2}\right)} \int_{h}^{\infty}\left(1+\frac{y^{2}}{v}\right)^{-\frac{v+1}{2}} T_{1}\left(\alpha y \sqrt{\frac{v+1}{y^{2}+v}} ; v+1\right) d y$ 
(ii)

$$
\begin{aligned}
\rho_{1}(h)= & \frac{1}{\pi}\left(1-\delta^{2}\right)\left(1+\frac{h^{2}}{v\left(1-\delta^{2}\right)}\right)\left(1+\frac{h^{2}}{v}\right)^{-\frac{v+1}{2}} \\
& \times I_{1}\left(\alpha h \sqrt{\frac{v+1}{v+h^{2}}} ; v+1\right)
\end{aligned}
$$

(iii) $\rho_{2}(h)=\frac{2}{(2 \pi)^{3 / 2}} \frac{\left(1-\delta^{2}\right)^{\frac{1}{2}} \Gamma\left(\frac{v+1}{2}\right)}{\left(\frac{v}{2}\right)^{\frac{1}{2}} \Gamma\left(\frac{v}{2}\right)} h\left(1+\frac{h^{2}}{v\left(1-\delta^{2}\right)}\right)\left(1+\frac{h^{2}}{v}\right)^{-\frac{v+1}{2}}$

$$
\begin{aligned}
& \times T_{1}\left(\alpha h \sqrt{\frac{v+1}{h^{2}+v}} ; v+1\right) \\
& \times\left[1-2 \delta\left(1-\delta^{2}\right)^{\frac{1}{2}}\left(\frac{v}{\pi}\right)^{\frac{1}{2}} \frac{\Gamma\left(\frac{v}{2}\right)}{\Gamma\left(\frac{v+1}{2}\right) h}\left(1+\frac{h^{2}}{v\left(1-\delta^{2}\right)}\right)^{-\frac{1}{2}}\right] .
\end{aligned}
$$

where $\alpha=\delta / \sqrt{1-\delta^{2}}$.

Proof. The proof of the proposition is based on the theorems and the lemmas reported in the literature. ${ }^{[4,10,15]}$

Let us consider the random field $Y$ as defined in (4.3), $Y$ satisfies the regularity conditions (i.e., $Y$ is twice differentiable inside $S$ and on the boundaries of $S$ ), with the restriction to the condition that $Y$ has the degree of freedom $v>2$.

For simplicity, $Y$ will be assumed centered, $\mu_{Y}=0$, with unit variance $\sigma_{Y}^{2}=1$ and skewness index $\delta>0$. However, in the case that $Y$ has a negative skewness, $\delta<0$, one can transform $Y$ to have a positive skewness dealing with $-Y$.

Since the EC densities, $\rho_{k}(h)$, in (5.3) do not depend on $\Lambda$, they will be calculated using the direct formulae in (5.8), assuming $Y$ is normalized such that $\Lambda=I_{d}$.

Using the conditional expectations, and conditioning on $U_{0}, Y$ becomes a Student's $\iota$ random field with $v$ degrees of freedom. So, the expected EC densities can be expressed as follows:

$$
\begin{aligned}
\rho_{k}(h)= & (-1)^{k-1} \int_{0}^{\infty}\left\{\mathbb{E}\left(\dot{Y}_{k}^{+} \operatorname{det}\left(\ddot{Y}_{\mid k-1}\right) \dot{Y}_{k-1}=0, Y=h, U_{0} \mid=u\right)\right. \\
& \left.\times p_{\mid k-1}(0 ; u, h)\right\} p_{U_{0} \mid}(u) d u \times p_{Y}(h),
\end{aligned}
$$

where $p_{\left|U_{0}\right|}(u)$ is given by:

$$
p_{U_{0} \mid}(u)=2 \frac{\Gamma\left(\frac{v+1}{2}\right)}{\sqrt{v \pi} \Gamma\left(\frac{v}{2}\right)}\left(1+\frac{y^{2}}{v}\right) d y
$$


The first- and second-order partial derivatives of $Y$ are expressed in terms of independent random variables (based on worsley, ${ }^{[21]}$ Lemmas 5.1 and 3.2), as follows:

$$
\begin{aligned}
\dot{Y}= & -\delta U_{0} V^{-1 / 2} z_{1}+v^{1 / 2} \sqrt{1-\delta^{2}}\left(1+\frac{\left(Y-\delta U_{0} \mid\right)^{2}}{v\left(1-\delta^{2}\right)}\right) W^{-1 / 2} z_{2} \\
\ddot{Y}= & \delta U_{0} V^{-1}\left\{-\left(Q-2 z_{1} z_{1}^{l}\right)-V+V^{1 / 2} H_{1}\right\} \\
& +\sqrt{1-\delta^{2}} v^{1 / 2}\left(1+\frac{\left(Y-\delta \mid U_{0}\right)^{2}}{v\left(1-\delta^{2}\right)}\right) W^{-1} \\
& \times\left\{-v^{-1 / 2}\left(1-\delta^{2}\right)^{-1 / 2}\left(Y-\delta \mid U_{0}\right)\left(P-2 z_{2} z_{2}^{l}\right)-z_{2} z_{3}^{l}-z_{3} z_{2}^{l}+W^{1 / 2} H_{2}\right\},
\end{aligned}
$$

where $z_{1}, z_{2}, z_{3} \sim \operatorname{Normal}_{d}(0, I), Q, P \sim$ Wisharl $_{d}(I, v-1), V \sim \chi_{v}^{2}, W \sim \chi_{v+1}^{2}$, $H_{1} \sim \operatorname{Normal}_{d \times d}(0, M), H_{2} \sim \operatorname{Normal}_{d \times d}(0, M)$, with covariance matrix $M$ defined in Adler, ${ }^{[2]}$ are all independent.

Conditioning on $U_{0} \mid, V, W, Y=h$, and on $\dot{Y}_{\mid k-1}=0$, and applying the expectations over both $V$ and $W,(5.10)$ becomes

$$
\begin{aligned}
\rho_{k}(h)= & (-1)^{k-1} \mathbb{E}_{\left|U_{0}\right|}\left\{\mathbb { E } _ { S , V } \left\{\mathbb{E}\left(\dot{Y}_{k}^{+} \operatorname{det}\left(\ddot{Y}_{k-1}\right) \dot{Y}_{\mid k-1}=0, Y=h, V, W, U_{0}=u\right)\right.\right. \\
& \left.\left.\times p_{k-1}(0 ; W, V, u, h)\right\}\right\} p_{\mid U_{0}}(u),
\end{aligned}
$$

where the components $\dot{Y}_{k}$ and $\ddot{Y}_{\mid k-1}$ are both independent under the conditional expectations. Thus, the term $\mathbb{E}\left(\dot{Y}_{k}^{+} \operatorname{det}\left(\ddot{Y}_{k-1}\right) \dot{Y}_{k-1}=0, Y=\right.$ $\left.h, V, W, U_{0}=u\right)$ in (5.13), can be expressed as follows:

$$
\begin{aligned}
\mathbb{E}\left(\dot{Y}_{k}^{+} \operatorname{det}\left(\ddot{Y}_{\mid k-1}\right)\left|\dot{Y}_{\mid k-1}=0, Y, V, W,\right| U_{0}\right) \\
=\mathbb{E}\left(\dot{Y}_{k}^{+} \dot{Y}_{\mid k-1}=0, Y, V, W, U_{0} \mid\right) \\
\quad \times \mathbb{E}\left(\operatorname{det}\left(\ddot{Y}_{\mid k-1}\right)\left|\dot{Y}_{\mid k-1}=0, Y, V, W,\right| U_{0}\right)
\end{aligned}
$$

Conditioning on $Y, V, W$, and $\mid U_{0}, \dot{Y}$ is a Gaussian random field and

$$
\begin{aligned}
& \mathbb{E}\left(\dot{Y}_{k}^{+} \dot{Y}_{\mid k-1}=0, Y, V, W, U_{0}\right) \\
& \quad=\frac{1}{\sqrt{2 \pi}}\left(\delta^{2} U_{0}^{2} V^{-1}+v\left(1-\delta^{2}\right)\left(1+\frac{\left(Y-\delta U_{0}\right)^{2}}{v\left(1-\delta^{2}\right)}\right)^{2} W^{-1}\right)^{1 / 2}
\end{aligned}
$$

Furthermore, conditional on $\dot{Y}_{1}=0, \ldots, \dot{Y}_{k-1}=0, \quad \ddot{Y}_{k-1}$ can be expressed as follows:

$$
\ddot{Y}_{k-1}=a Q+b P+c H_{1}+d H_{2}+e
$$


where

$$
\begin{aligned}
& a=-\delta U_{0} \mid V^{-1} \\
& b=-\left(1+\frac{\left(Y-\delta U_{0} \mid\right)^{2}}{v\left(1-\delta^{2}\right)}\right) W^{-1}\left(Y-\delta U_{0} \mid\right) \\
& c=\delta U_{0} \mid V^{-1 / 2} \\
& d=\left(1+\frac{\left(Y-\delta U_{0} \mid\right)^{2}}{v\left(1-\delta^{2}\right)}\right) W^{-1 / 2} \\
& e=\delta U_{0} \mid
\end{aligned}
$$

Then, using Lemma (A.1) and Lemma (A.2), (in Appendix 8), due to Worsley ${ }^{[21]}$ we get:

$$
\begin{aligned}
\mathbb{E}\left(\operatorname{det}\left(\ddot{Y}_{\mid k-1}\right) \dot{Y}_{\mid k-1}=0, Y, V, W, U_{0}\right) \\
=\mathbb{E}\left(\operatorname{det}\left(a Q+b P+c H_{1}+d H_{2}+e\right)\right) \\
=\sum_{j=0}^{\left\lfloor\frac{k-1}{2}\right\rfloor} \sum_{l=0}^{k-1-2 j\rfloor} \sum_{n=0}^{\left\lfloor\frac{k-1-2 j-l}{2}\right\rfloor}\left(\begin{array}{c}
v-1 \\
l
\end{array}\right)\left(\begin{array}{c}
v-1 \\
k-1-2 j-l
\end{array}\right) a^{l} b^{k-1-2 j-l} \\
\quad \times \frac{(-1)^{n+j}(2 n) !(k-1) !}{2^{n+j}(n+j) !} c^{2 j} d^{2 n} b^{l} \times a^{k-1-2 j-l} e^{k-1-2 j-l-2 n}
\end{aligned}
$$

The joint probability density function, $p_{\mid k-1}(0 ; V, W, u, h)$, of the first $k-1$ components of $\dot{Y}$ conditioning on $V, W, U_{0}=u$ and on $Y=h$ is then:

$$
\begin{aligned}
& p_{k-1}(0 ; V, W, u, h) \\
& =(2 \pi)^{-\frac{k-1}{2}}\left(\delta^{2} u^{2} V^{-1}+v\left(1-\delta^{2}\right)\left(1+\frac{(h-\delta u)^{2}}{v\left(1-\delta^{2}\right)}\right)^{2} W^{-1}\right)^{-\frac{k-1}{2}}(5
\end{aligned}
$$

So,

$$
\begin{aligned}
\mathbb{E}\left(\dot{Y}_{k}^{+} \operatorname{det}\left(\ddot{Y}_{\mid k-1}\right)\right. & \left.\dot{Y}_{\mid k-1}=0, Y=h, V, W, U_{0}=u\right) p_{k-1}(0 ; W, V, u, h) \\
= & (2 \pi)^{-\frac{k}{2}} \times\left(\delta^{2} u^{2} V^{-1}+v\left(1-\delta^{2}\right)\left(1+\frac{(h-\delta u)^{2}}{v\left(1-\delta^{2}\right)}\right)^{2} W^{-1}\right)^{-\frac{k}{2}+1} \\
& \times \sum_{j=0}^{\left\lfloor\frac{k-1}{2}\right\rfloor} \sum_{l=0}^{k-1-2 j\left\lfloor\frac{k-1-2 j-l}{2}\right\rfloor} \sum_{n=0}^{2}\left(\begin{array}{c}
v-1 \\
l
\end{array}\right)\left(\begin{array}{c}
v-1 \\
k-1-2 j-l
\end{array}\right)
\end{aligned}
$$




$$
\begin{aligned}
& \times \frac{(-1)^{k-1-j+n}(2 n) !(k-1) !}{2^{n+j}(n+j) !}\left(\delta^{2} u^{2}\right)^{k-1-l-j-n} v^{n}\left(1-\delta^{2}\right)^{n} \\
& \times\left(1+\frac{(h-\delta u)^{2}}{v\left(1-\delta^{2}\right)}\right)^{2 n+l}(h-\delta u)^{l} W^{-(n+l)} V^{-k+1+j+l}
\end{aligned}
$$

By computing the expectation over $W$ and $V$ in the last equation, where $\mathbb{E}\left[V^{j}\right]=2^{j} \Gamma(v / 2+j) / \Gamma(v / 2)$ and $\mathbb{E}\left[W^{j}\right]=2^{j} \Gamma((v+1) / 2+j) / \Gamma((v+1) / 2)$, as well integrating over the density of $\mid U_{0}$ and multiplying by the density of $Y$, we obtain $\rho_{k}(h)$.

The density of $Y, p_{Y}(h)$, at $h$ is a skew Student's $t$ probability density with $v$ degrees of freedom such that:

$$
p_{Y}(h)=\frac{\Gamma\left(\frac{v+1}{2}\right)}{\sqrt{v \pi} \Gamma\left(\frac{v}{2}\right)}\left(1+\frac{h^{2}}{v}\right)^{-\frac{v+1}{2}} T_{1}\left(\alpha h \sqrt{\frac{v+1}{h^{2}+v}} ; v+1\right)
$$

Then, for $k=1$ :

$$
\rho_{1}(h)=\frac{1}{\pi}\left(1-\delta^{2}\right)\left(1+\frac{h^{2}}{v\left(1-\delta^{2}\right)}\right)\left(1+\frac{h^{2}}{v}\right)^{-\frac{v+1}{2}} T_{1}\left(\alpha h \sqrt{\frac{v+1}{v+h^{2}}} ; v+1\right)
$$

and for $k=2$, we obtain:

$$
\begin{aligned}
\rho_{2}(h)= & \frac{2}{(2 \pi)^{\frac{3}{2}}}\left(\frac{\left(1-\delta^{2}\right)^{\frac{1}{2}} \Gamma\left(\frac{v+1}{2}\right)}{\left(\frac{v}{2}\right)^{\frac{1}{2}} \Gamma\left(\frac{v}{2}\right)} h\right)\left(1+\frac{h^{2}}{v\left(1-\delta^{2}\right)}\right)\left(1+\frac{h^{2}}{v}\right)^{-\frac{v+1}{2}} \\
& \times I_{1}\left(\alpha h \sqrt{\frac{v+1}{h^{2}+v}} ; v+1\right) \\
& \times\left[1-2 \delta\left(1-\delta^{2}\right)\left(\frac{v}{\pi}\right)^{\frac{1}{2}} \frac{\Gamma\left(\frac{v}{2}\right)}{\Gamma\left(\frac{v+1}{2}\right) h}\left(1+\frac{h^{2}}{v\left(1-\delta^{2}\right)}\right)^{-\frac{1}{2}}\right]
\end{aligned}
$$

where $\alpha=\delta / \sqrt{1-\delta^{2}}$. For $j=0, \rho_{0}(h)=\mathscr{L}_{0}(S) \mathbb{P}[Y \geq h],{ }^{|22|}$ where $\mathscr{L}_{0}(S)=$ $\chi(S)$ is the Euler-Poincaré characteristic of the subset $S$. In our case, $\mathscr{L}_{0}(S)=1$.

Corollary 5.3.1. The expected Euler-Poincaré characleristic, $\mathbb{E}\left[\chi\left(E_{h}(Y, S)\right)\right]$, of the skew Student s $t$ excursion sets, $E_{h}(Y, S)$, at high thresholds, $(h \rightarrow \infty)$, becomes

$$
\mathbb{E}\left[\chi\left(E_{h}(Y, S)\right)\right]=2 R_{1} R_{2}\left(\frac{\sqrt{\operatorname{del}(\Lambda)}}{(2 \pi)^{3 / 2}}\right)\left(\frac{\left(1-\delta^{2}\right)^{\frac{1}{2}} \Gamma\left(\frac{v+1}{2}\right)}{\left(\frac{v}{2}\right)^{\frac{1}{2}} \Gamma\left(\frac{v}{2}\right)}\right)
$$




$$
\begin{aligned}
& \times h\left(1+\frac{h^{2}}{v\left(1-\delta^{2}\right)}\right)\left(1+\frac{h^{2}}{v}\right)^{-\frac{v+1}{2}} \\
& \times T_{1}\left(\alpha h \sqrt{\frac{v+1}{v+h^{2}}} ; v+1\right)
\end{aligned}
$$

The expected Euler-Poincaré characteristic of the skew Student's $t$ excursion set, $E_{h}(Y, S)$, can be expressed in terms of the known EulerPoincaré characteristic, $\mathbb{E}\left[\chi\left(E_{h}\left(T^{v}, S\right)\right)\right]$, of Student's $\ell$ excursion sets, such that:

$$
\begin{aligned}
& \mathbb{E}\left[\chi\left(E_{h}(Y, S)\right)\right] \\
& \cong \frac{2}{\left(1-\delta^{2}\right)} T_{1}\left(\alpha h \sqrt{\frac{v+1}{v+h^{2}}} ; v+1\right) \mathbb{E}\left[\chi\left(E_{h}\left(T^{v}, S\right)\right)\right]\left\{1+O\left(\frac{1}{h}\right)\right\}
\end{aligned}
$$

when $h \rightarrow \infty$.

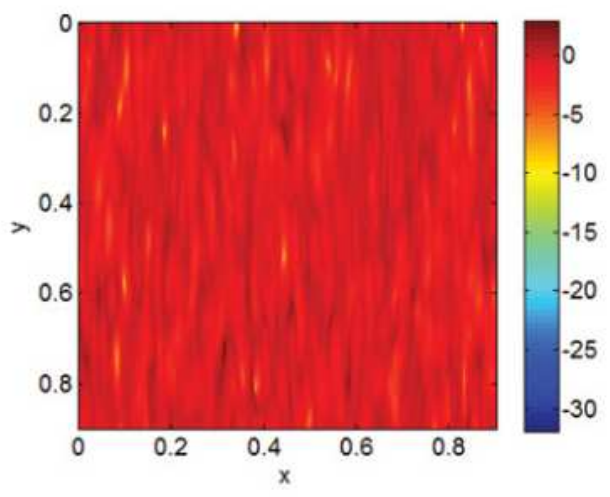

(a)
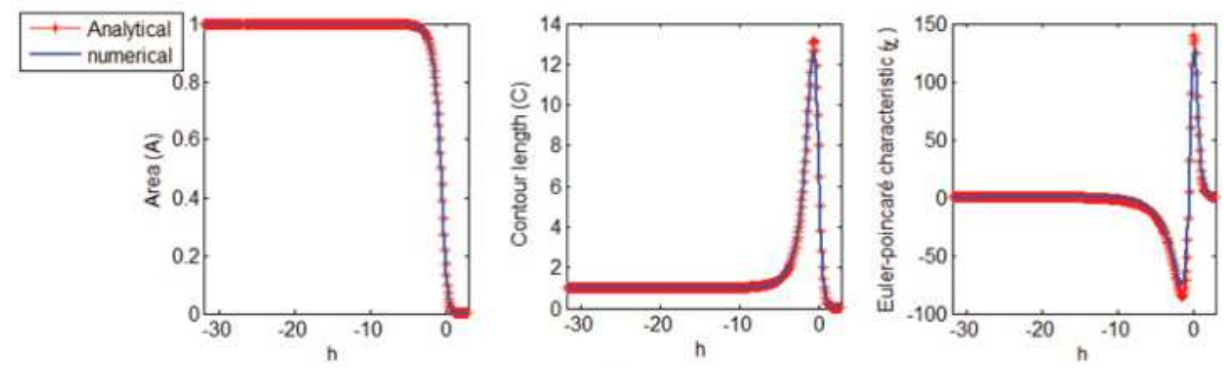

(b)

FIGURE 1 First simulation example: (a) anisotropic skew Student's $t$ random field with 5 degrees of freedom and skewness index $\delta=-0.9$ realized on a lattice of $500 \times 500$ points in $[0,1]^{2}$; (b) the numerical and the analytical Lipschitz-Killing curvatures, $A, C$, and $\chi$, respectively. (color figure available online). 


\section{SIMULATION RESULTS}

In this section, simulation examples of the skew Student's $t$ random fields are illustrated for positive (resp. negative) values of the skewness index $\delta$. An illustration of the analytical expressions of the $j$ th dimensional Lipschitz-Killing curvatures are given compared to the numerical ones that are computed from the simulations for validation. For this aim, 50 realizations of the skew Student's $t$ random field have been generated for two different examples. The simulations are investigated on a lattice of $500 \times 500$ points in both $x$ and $y$ directions within the unit square $[0,1] \times$ $[0,1]$. Figures $1(\mathrm{a})$ and 2 (a) illustrate two examples of stationary skew Student's $t$ random fields with 5 degrees of freedom and with skewness indexes $\delta=-0.9$ and $\delta=0.4$, respectively. Their analytical and numerical Lipschitz-Killing curvatures are represented in Figures 1(b) and 2(b), respectively.

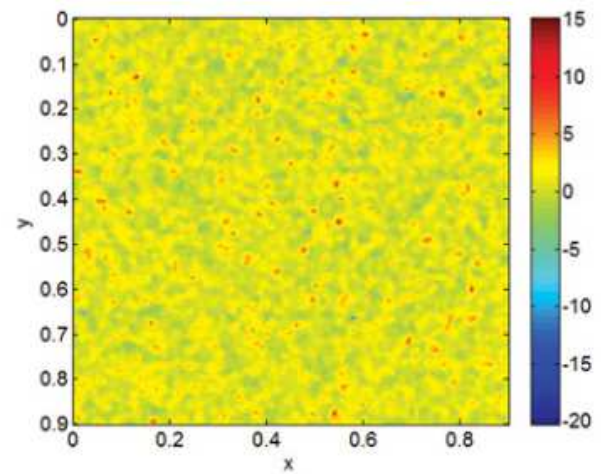

(a)
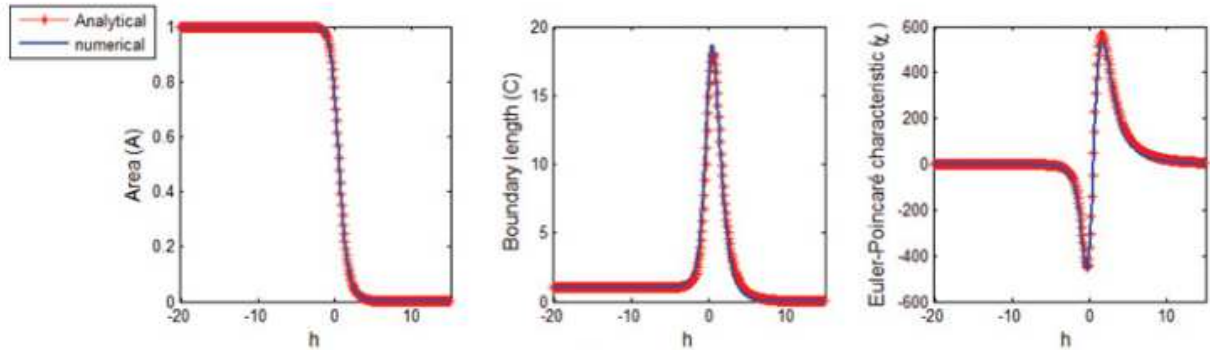

(b)

FIGURE 2 Second simulation example: (a) isotropic skew Student's $t$ random field with 5 degrees of freedom and skewness index $\delta=0.4$ realized on a lattice of $500 \times 500$ points in the unit square $[0,1]^{2}$; (b) the numerical and the analytical Lipschitz-Killing curvatures, $A, C$, and $\chi$, respectively. (color figure available online). 


\section{APPLICATION}

The stochastic model has been tested on a real 3D microstructured rough surface of a (ultra-high-molecular-weight polyethylene) component. ${ }^{[12]}$ The surface has been measured by a noncontact white light interferometry (Bruker nanoscope Wyko NT 9100), on a lattice of $480 \times 640$ points with a spatial resolution equal to $1.8 \mu \mathrm{m}$ in both $x$ and $y$ directions; see Figure 3(a). There are two main reasons for using the expected Euler-Poincaré characteristic in such applications: firstly, at high threshold, it counts the number of connected components that approximate the number of the local maxima and minima of $Y$ such

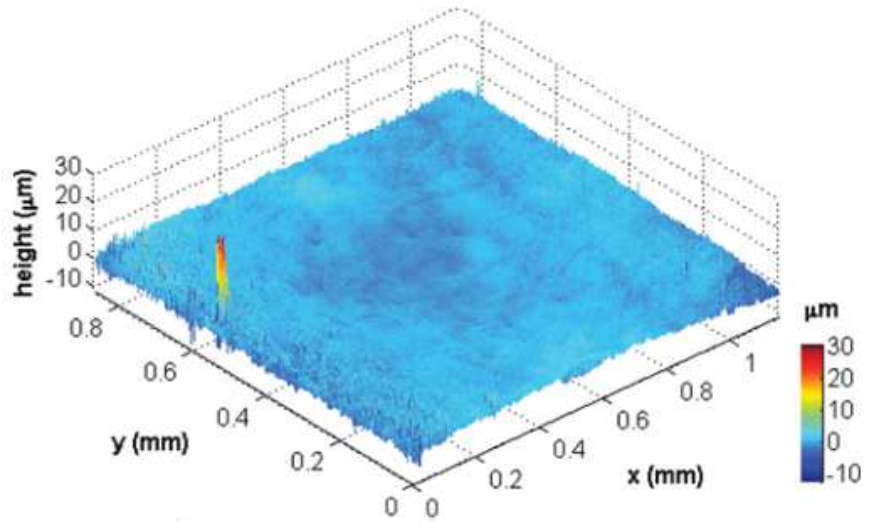

(a)

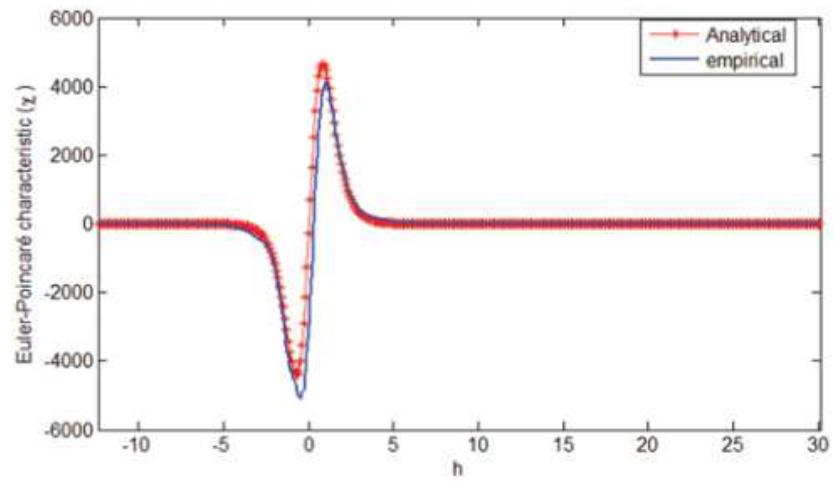

(b)

FIGURE 3 An application example: (a) a real $3 \mathrm{D}$ surface roughness topography digitized on a lattice of 480 points with spatial sampling steps equal to $1.8 \mu \mathrm{m}$ in $x$ and $y$ directions; (b) fitting the empirical and the analytical Euler-Poincare characteristic functions of the real surface upcrossings and the skew Student's $t$ excursion sets for 10 degrees of freedom and skewness index $\delta=0.7$. (color figure available online). 
that ${ }^{[2,14]}$ :

$$
\begin{aligned}
& \mathbb{P}\left[Y_{\max }>h\right] \cong \mathbb{E}\left[\chi\left(E_{h}(Y, S)\right)\right] \\
& \mathbb{P}\left[Y_{\min }<h\right] \cong \mathbb{E}\left[\chi\left(E_{-h}(-Y, S)\right)\right]
\end{aligned}
$$

when $(h \rightarrow \infty)$.

Thus it can describe its roughness. Secondly, its explicit formulae enable estimating the random field's parameters, or estimating, for a specific test of significance, the threshold $h$ that can detect whether some materials of the surface are lost.

The results presented in this article concern fitting the surface roughness stochastic model with the real 3D heights map using the EulerPoincaré characteristic function. Figure $3(\mathrm{~b})$ shows the analytical and numerical Euler-Poincaré characteristic of the skew Student's $t$ excursion sets and the real surface upcrossings, respectively, for 10 degrees of freedom and skewness index $\delta=0.7$.

The results show the ability to use this stochastic model for studying the morphology and size distribution of the excursion sets at certain significant thresholds in order to describe the functionality of the surface and to determine its performance before and maybe after involving it in the hip replacement surgery.

\section{CONCLUSION}

A special class of skew Student's $\iota$ random fields with $v$ degrees of freedom has been introduced and studied geometrically. We derived, an analytical formulae of the Euler characteristic densities of the skew Student's $l$ excursion sets in order to estimate their Lipschitz-Killing curvatures on a rectangular subset $S \subset \mathbb{R}^{d},(d=2)$, and they can be extended to higher dimensions $d>2$.

Simulations have been performed to validate the analytical formulae. Then, an application of modeling the roughness topography of a worn engineering surface has been investigated. The results are promising and show the ability of using the skew Student's $t$ random field for describing the surface roughness changes between the different spatial samples and/or during a specific temporal process, which is the aim of our future work.

\section{APPENDIX}

Lemma A.1 (Ref.. $\left.{ }^{121}\right)$. Let $H \sim \operatorname{Normal}_{d \times d}(0, M)$ and let $B$ be a fixed symmetric $d \times d$ matrix. Then:

$$
\mathbb{E}[\operatorname{det}(B+H)]=\sum_{j=0}^{\lfloor d / 2\rfloor} \frac{(-1)^{j}(2 j) !}{2^{j}} \operatorname{detr}_{d-2 j}(B)
$$


where $\operatorname{det}_{k}(B)$ stands for the sum of the determinant of all $k \times k$ principal minors of $B$.

Lemma A.2 (Ref. $\left.{ }^{[21]}\right)$. Let $Q \sim \operatorname{Wishart}\left(I_{d}, v\right), \quad P \sim \operatorname{Wishart}\left(I_{d}, \mu\right), \quad H \sim$ Normal $_{d \times d}(0, M)$ be independent and let $a, b$ be fixed scalars. Then:

$$
\mathbb{E}[\operatorname{del}(P+a Q+b H)]=\sum_{j=0}^{\lfloor d / 2\rfloor} \frac{(-1)^{j}}{2^{j} d !} b^{2 j} \sum_{k=0}^{d-2 j}\left(\begin{array}{l}
v \\
l
\end{array}\right)\left(\begin{array}{c}
\mu \\
d-2 j-l
\end{array}\right) a^{k}
$$

\section{REFERENCES}

1. Abrahamsen, P. A review of Gaussian random fields and correlation functions. Norwegian Computing Center, Tech. Rep. 917; Norwegian Computing Center: Oslo, Norway, April 1997.

2. Adler, R.J. The Geometry of Random Fields (Wiley Series in Probability and Statistics). John Wiley \& Sons: Chichester, UK, 1981.

3. Adler, R.J.; Taylor, J. Random Fields and Geometry. 1st ed; Springer: Berlin, 2007.

4. Adler, R.; Taylor, J. Topological Complexity of Smooth Random Functions. Saint Flour Lectures, July 2009.

5. Adler, R.J.; Taylor, J.E.; Worsley, K.J. Applications of random fields and geometry: Foundations and case studies," 2007, in preparation. Available at http://ie.technion.ac.il/ radler/publications.html (accessed 2011).

6. Alodat, M.T.; Al-Rawwash, M.Y. Skew-Gaussian random field. J. Comput. Appl. Math. 2009, 232 (2), 496-504.

7. Azzalini, A.; Capitanio, A. Statistical applications of the multivariate skew normal distribution. J. Roy. Stat. Soc. Ser. B 1999, 61 (3), 579-602.

8. Azzalini, A.; Capitanio, A. Distributions generated by perturbation of symmetry with emphasis on a multivariate skew $t$-distribution. J. Roy. Stat. Soc. Ser. B 2003, 65 (2), 367-389.

9. Basso, R.M.; Lachos, V.H.; Cabral, C.R.B.; Ghosh, P. Robust mixture modeling based on scale mixtures of skew-normal distributions. Computat. Stat. Data Anal. 2010, 54 (12), 2926-2941.

10. Cabral, C.R.B.; Lachos, V.H.; Prates, M.O. Multivariate mixture modeling using skew-normal independent distributions. Computat. Stat. Data Anal. 2012, 56 (1), 126-142.

11. Genton, M.G. Skew-Elliptical Distributions and Their Applications: A Journey Beyond Normality; Taylor \& Francis: London, 2004.

12. Geringer, J.; Boyer, B.; Farizon, F. Understanding the dual mobility concept for total hip arthroplasty. investigations on a multiscale analysis-highlighting the role of arthrofibrosis. Wear 2011, 271 (9-10), 2379-2385.

13. Hadwiger, II. Vorlesungen über Inhalt, Oberfläche und Isoperimetrie 93 (1), Springer-Verlag: Berlin, Heidelberg, 1957.

14. Hasofer, A.M. Upcrossings of random fields. Adv. Appl. Probab. 1978, 10, 14-21.

15. Jones, M.C. The $t$ family and their close and distant relations. J. Kor. Stat. Soc. 2008, 37 (4), 293-302.

16. Lachos, V.H.; Labra, F.V.; Bolfarine, H.; Ghosh, P. Multivariate measurement error models based on scale mixtures of the skewnormal distribution. Statistics 2010, 44 (6), 541-556.

17. Morse, M.; Cairns, S.S. Critical Point Theory in Global Analysis and Differential Topology; Vol. 33: An Introduction. 1st ed; Academic Press: New York, 1969.

18. Santalo, L.A. Integral Geometry and Geometric Probability, Cambridge University Press: Cambridge, UK, 2004.

19. laylor, J.E. Random fields of multivariate test statistics, with applications to shape analysis. Ann. Stat. $200836(1), 1-27$. 
20. Taylor, J; Adler, R. Euler characteristic for gaussian ficlds on manifolds. Ann. Probab. 2003, 31 (2), 533-563.

21. Worsley, K.J. Local maxima and the expected euler characteristic of excursion sets of $\chi^{2}$, $\mathrm{f}$ and t fields. Adv. Appl. Probab. 1994, 26 (1), 13-42.

22. Worsley, K. Boundary corrections for the expected euler characteristic of excursion sets of random fields, with an application to astrophysics. Adv. Appl. Probab. 1995, 27, 943-959.

23. Yaglom, A.M. Correlation Theory of Stationary and Related Random Functions: Vol. I: Basic Results, 1st ed; Springer: Berlin, 1987. 